\title{
组合化学在开发纳米材料以及纳米生物医学研究中的应用进展
}

\author{
吴金梅苏高星张斌间兵* \\ (山东大学化学与化工学院 济南 250100)
}

\begin{abstract}
摘要 组合化学是一种将计算机辅助设计、有机化学合成以及高通量篮选一体化的技术. 它以高效、微量、高度自动 化的特点而受到世人瞩目, 对药物研发中加速寻找先导化合物起到了极大的推进作用. 近年来随着纳米技术的迅速发 展, 组合化学策略和高通量技术也在此领域中得到应用. 为在今后的研究中能够更好地将组合化学技术应用于纳米材 料和纳米技术研究领域, 本文综述了组合化学在开发新型纳米材料以及通过对纳米材料进行表面化学修饰来提高其在 生物医学领域的应用研究进展, 并对目前应用于纳米技术研究的高通量笁选技术, 如磁共振成像、自动化基因芯片系 统和荧光激活细胞分类术以及对纳米组合化学目前遇到的一些挑战进行了简单概括, 并对其未来的发展趋势提出了展 望.
\end{abstract}

关键词 组合化学; 高通量篮选; 纳米材料; 纳米医学; 纳米组合化学

\section{Nanocombinatorial Chemistry in Nanomaterial Discovery and Nanomedicine}

\author{
Wu, Jinmei Su, Gaoxing Zhang, Bin Yan, Bing* \\ (School of Chemistry and Chemical Engineering, Shandong University, Jinan 250100)
}

\begin{abstract}
Combinatorial chemistry is a scientific technology covering computational chemistry, organic synthesis, and high-throughput screening. Combinatorial chemistry can achieve synthesis and screenings of large compounds within a short time, which can greatly improve compounds synthesis efficiency, shorten compounds discovery cycle and reduce cost effectively. It has greatly impacted on lead compounds discovery with high efficiency, miniaturization and high automation. In recent years, combinatorial chemistry was not only applied in drugs discovery, but also applied in catalyst discovery, pesticide discovery and polymer discovery because of its advantages. With the rapid development of nanotechnology, combinatorial chemistry strategies and high throughput technologies have been introduced to this area. The development of nanotechnology will have tremendous impacts on every aspect of our society. However, it requires a time-consuming research effort on the discovery and optimization of novel nanomaterials with unique properties. Parallel reactions and high-throughput screening are considered to be more efficient than conventional linear operations. In addition, combinatorial chemistry can achieve the biological activities variety of nanomaterials by modifying nanomaterials surface. In this review, we summarized the applications of nanocombinatorial chemistry in the discovery of novel nano materials and the discovery of medicines with modified nanomaterials. We also summarized many high-throughput screening technologies which were used in nanotechnology research, including magnetic resonance imaging (MRI), automation gene chip system and fluorescent activated cell sorter (FACS). Finally, for easily understanding the tendency of nano-combinatorial chemistry, some challenges and future perspectives of nanocombinatorial chemistry were also included.
\end{abstract}

Keywords combinatorial chemistry; high-throughput screening; nanomaterial; nanomedicine; nanocombinatorial chemistry

\section{1 引言}

组合化学是一种将计算机辅助分子设计、有机化学 合成及自动化和高通量篮选评价一体化的技术, 主要由 三部分组成, 即组合库的合成、库的分析表征和库的篮 选. 相对于传统方法把大量注意力放在研究较少的化学 多样性空间中的分子结构细节上, 组合化学则强调首先 快速地搜索尽可能大的化学多样性空间. 利用组合化学 技术可以在短时间内实现大批量化合物的合成以及篮
选，不仅可以大大提高化合物的合成效率，还缩短了研 究周期以及降低实验成本. 目前, 组合化学已经在研发 新型药物 ${ }^{[1]}$ 、催化剂 ${ }^{[2]}$ 、聚合物 ${ }^{[3]}$ 和新农药 ${ }^{[4]}$ 等领域中得 到了广泛应用.

纳米技术, 是指在 $0.1 \sim 100 \mathrm{~nm}$ 的尺度范围研究电 子、原子和分子内的运动规律和特性的一门技术，它的 出现从根本上改变人类社会对多种不可持续的传统工 业技术的依赖，并将大大推动在能源、电子、信息以及 医学领域内的革命性飞跃 ${ }^{[5,6]}$. 由于纳米材料具有优良

*E-mail: drbingyan@yahoo.com; Tel.: 0531-88366257; Fax: 0531-88380029

Received January 17, 2013; published March 1, 2013.

Project supported by the National Basic Research Program of China (973 Program, No. 2010CB933504) and the National Natural Science Foundation of China (Nos. 21137002, 21077068).

项目受国家重点基础研究发展计划(973 计划)项目(No. 2010CB933504)和国家自然科学基金(Nos. 21137002, 21077068)资助. 
的电学性质、光学性质和生物相容性, 使纳米技术得到 迅速发展, 已渗透于生物医学、传感器、微型电子器件 等重要技术领域中. 然而, 对于如何能够快速建立一些 具有独特性质的纳米材料库以及如何在一个巨大的纳 米材料库中快速发现目标纳米材料仍然面临着巨大的 挑战. 采用传统模式来实现纳米材料库的合成和篎选会 耗费很多的人力以及时间. 近年来, 随着纳米技术的迅 速发展, 纳米组合化学这一概念首次被提出 ${ }^{[7]}$, 并已成 功将组合化学应用于构建纳米材料组合库以及纳米生 物医学研究. 采用组合化学高通量平行合成和笁选的技 术手段能够有效地缩短发现目标纳米材料的过程. 我们 在前期研究中利用纳米组合化学策略合成出碳纳米管、 金纳米颗粒等多个纳米材料化合物库, 并建立了相关的 分析表征及生物管选方法, 通过构效分析, 发现纳米材 料表面化学与多样性修饰能够降低纳米材料自身的毒 性、增强其特异识别蛋白质能力, 显示了纳米组合化学 在发现新型纳米材料以及在纳米生物医药领域中的应 用中发挥着重要作用.

本文将从三个方面对近年来组合化学在开发纳米 材料以及纳米生物医学研究中的应用进展进行总结. 第 一, 关于组合化学技术在开发新型纳米材料领域中的应 用. 例如利用组合化学方法建立纳米金属颗粒催化剂库 来优化碳纳米管的生长条件; 第二, 通过组合化学技术 对纳米材料表面进行功能化修饰改进其生物相容性, 并 通过特定官能团修饰赋予其特殊的功能, 进而提高其生 物医学应用价值, 如提高肿瘤细胞靶向性, 肿瘤诊断等; 第三, 介绍近年来应用于纳米技术研究中的高通量篎选 技术, 并对纳米组合化学的发展提出了展望.

\section{2 组合化学在开发新型纳米材料中的应用}

随着纳米材料被广泛应用于多个领域, 人们对纳 米材料自身性质的要求也越来越高, 许多研究学者正致 力于开发出一些具有独特性质的新型纳米材料. 组合化 学策略由于其快速、高效的特点而被引进用于开发新型 纳米材料, 目前已经成功应用于纳米催化剂库的构建以 及聚合物纳米颗粒库的构建等. 同时, 高通量技术也成 功应用于单层石墨烯制备过程中.

\section{1 纳米催化剂组合库的建立}

纳米材料由于具有尺寸小、比表面积大、表面能高 和表面催化中心多等独特性质而为其成为催化剂提供 了必要条件. 近年来, 许多研究结果表明了纳米材料在 催化剂领域具有优异的催化活性和选择性. 纳米金属将 金属独特的物化性质和纳米材料的特殊性质有机结合 在一起, 已逐步应用于加氢 ${ }^{[8]}$ 、氧化 ${ }^{[9]}$ 、耦合 ${ }^{[10]}$ 等反应, 同时也可以用来催化其他纳米材料如碳纳米管的生长. 鉴于组合化学技术对于建立和篮选目标物的高效性, 研 究者利用它来合成并篮选出特定反应所适合的纳米金
属催化剂. Senkan 等 ${ }^{[11]}$ 利用高通量脉冲激光烧蚀方法 (High Throughput pulsed laser ablation, HT-PLA)在多种 基底 $\left(\mathrm{g}-\mathrm{Al}_{2} \mathrm{O}_{3}, \mathrm{CeO}_{2}, \mathrm{TiO}_{2}, \mathrm{SiO}_{2}\right.$ 和 $\left.\mathrm{Y}-\mathrm{ZrO}_{2}\right)$ 上合成出多种 不同的金属纳米催化剂，其中包括金属铑、铑/铂二元金 属和铑/铂/金三元金属催化剂. 他们通过丙烯的氧化效 果对库中的金属纳米催化剂进行催化效果篮选. 利用丙 烯的氧化产物氧化丙烯(PO)和丙酩 (AT)的量来反映丙 烯的氧化效果(图 1). 实验结果发现, 在 $\mathrm{TiO}_{2}$ 基底上负 载 $0.004 \%$ 铑纳米材料, $275{ }^{\circ} \mathrm{C}$ 条件下, PO 和 AT 的总产 量为 $13 \%$, 此时对丙烯氧化反应具有最好的催化效果. 同时他们还利用 HT-PLA 方法发现，在多元金属催化剂 的制备过程中，活性金属的沉积序列会对催化剂催化活 性和选择性的形成产生重要影响 ${ }^{[12]}$. 组合纳米催化剂 库的建立可以大大缩短制备纳米催化剂时探讨影响因 素的时间, 为未来开发更多特定反应中的纳米催化剂带 来很大的推动作用.

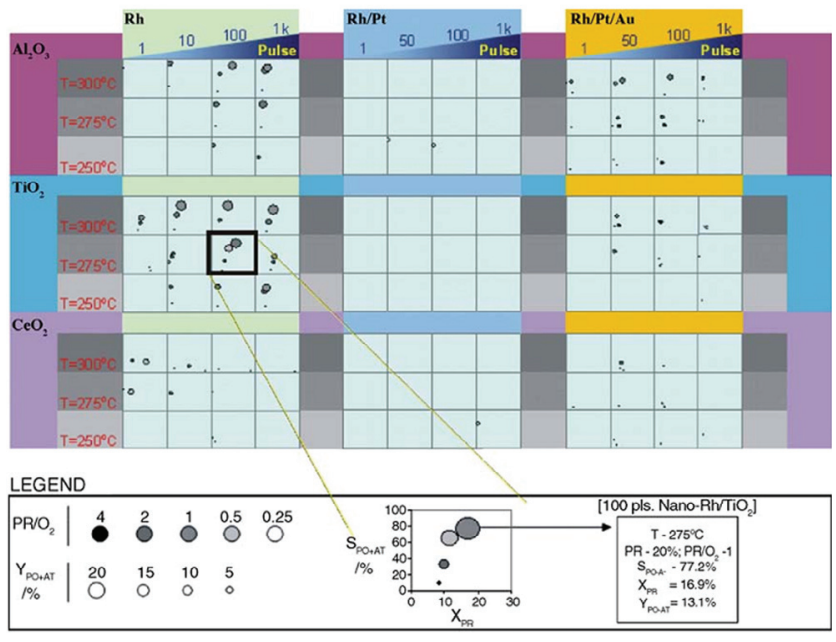

图 1 金属纳米催化剂库的笁选结果. 每个格子中圆圈或斑点的大小 代表 PO 和 AT 产量高低; 不同圆圈代表的是实验中 $\mathrm{C}_{2} \mathrm{H}_{6}$ 与 $\mathrm{O}_{2}$ 的比例. 从参考文献[11]允许转载, 2006 Elsevier 版权所有.

Figure 1 Selected results of metal nanoparticle catalysises. The sizes of the circles or spots (see legend) presented $\mathrm{PO}+\mathrm{AT}$ yields. Different circles in each square correspond to different $\mathrm{C}_{2} \mathrm{H}_{6} / \mathrm{O}_{2}$ ratios used in the experiments, in accordance with the gray scale shown in the legend.

近年来, 金属纳米颗粒催化剂也被广泛应用于催化 碳纳米管的生长 ${ }^{[13 \sim 16]}$. Noda 等 ${ }^{[13]}$ 利用组合掩膜沉积法 建立了一个 $\mathrm{Co}$ 纳米颗粒催化剂库, $\mathrm{SiO}_{2}$ 基底上的 $\mathrm{Co}$ 亚 单原子层会自发形成 Co 纳米粒子, 这些 Co 纳米粒子可 以催化生长出高质量的单壁碳纳米管(SWCNTs). 同时, 这种组合掩膜沉积法也可应用于合成篎选二元金属催 化剂库. 通过在 $\mathrm{SiO}_{2} / \mathrm{Si}$ 基底上制备优化不同公称厚度 的 $\operatorname{Co}(0.2 \sim 8 \mathrm{~nm})-\mathrm{Mo}(0.2 \sim 4 \mathrm{~nm})$ 二元金属催化剂库，同 时通过乙醇催化化学气相沉积来控制 SWCNTs 的生 $k^{[14]}$. 因此, 组合掩膜沉积法将成为开发非均相催化剂 的有利手段. 


\section{2 聚合物纳米颗粒组合库的建立}

阳离子聚合物由于没有免疫原性, 可根据需求灵活 设计其分子结构, 可通过控制粒径和表面性质来控制载 体的生物效应等优点而在基因治疗中受到青睐. 由于组 合化学可以将合成、加工过程简单化, 并且可以同时实 现对纳米材料多种组合性质的改变与管选 ${ }^{[17]}$. 因此, 它 在寻找具有高效基因转载效率的聚合物纳米颗粒过程 中表现出很大的优势和潜力.

目前为止，已经有许多的文章报道了关于阳离子聚 合物组合库的建立以及对于组合库基因转染效率的评 价 $^{[18 ~ 20]}$. Anderson 等 ${ }^{[21]}$ 利用高通量半自动化方法建立 了一个含 2350 种结构独特、可降解的聚氨基脂类 (PBAEs)聚合物库. 经过细胞实验篮选后得到其中 46 种 聚合物的基因转染效率优于传统的非病毒型基因载体, 如聚乙烯亚胺(PEI). 另外, 研究发现改变 PBAEs 聚合 物的直链骨架, 侧链结构和封端基团会对 PBAEs 的基 因转染效率产生很大影响, 聚合物骨架和侧链基团疏水 性的增加可以提高 PBAEs 的基因转染效率 ${ }^{[22]}$. 同时, 封 端基团的改变可以有效改变 PBAEs 的基因转染效率. 通过加入不同封端基团可以建立不同末端修饰化的 PBAEs 库 ${ }^{[22 ~ 25]}$. 例如, Zugates 等 ${ }^{[18]}$ 建立了一个含有不 同封端基团的 PBAEs 库, 利用非洲绿猴肾细胞系 (COS-7 cells)通过苂光素酶的表达来评价它们的基因转 染效率, 研究结果表明当亲水性封端基团的末端带羟基 或伯氨基时可以有效提高聚合物的基因转染效率.

研究者还利用组合化学方法制备 PEI 聚合物纳米颗 粒库, Van Vliet 等 ${ }^{[19]}$ 通过对 PEI 进行修饰建立了一个含 435 种功能化的多样性的 PEI 聚合物库, 通过 DNA 结 合、细胞毒性和基因转染效率实验综合评价库中聚合物 的生物特性. 研究表明, 与未修饰的 PEI 相比, 一些修 饰化的 PEI 可以明显提高基因传染效率, 并对不同细胞 表现出不同的选择性. 细胞识别选择性的提高将对某些 特定癌症的基因治疗十分有利. 此外, Barua 等 ${ }^{[26]}$ 利用 二环氧甘油醚的环氧基团和聚胺类上的氨基之间的开 环聚合反应合成了一个阳离子聚合物组合库, 通过篮选 发现库中的一些阳离子聚合物与传统非病毒基因载体 PEI 相比, 表现出更优异的基因转染效果以及更低的细 胞毒性，可以为寻找更优良的基因载体作铺垫.

\section{3 其他纳米材料库的建立}

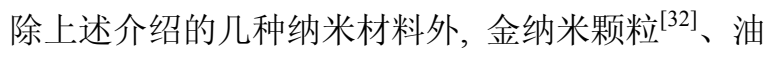
脂纳米材料 ${ }^{[33]}$ 、纳米薄膜 ${ }^{[34]}$ 和介孔二氧化硅纳米颗粒 ${ }^{[35]}$ 也都已实现高通量合成. Mulet 等 ${ }^{[33]}$ 利用两亲化合物, 通过调节两亲分子和药物各自的比例以及改变药物种 类, 高通量合成出一个载药的两亲油脂纳米晶体库. Tse 等 ${ }^{[35]}$ 则利用一个自动化机器平台技术高通量合成出用 于磁共振成像的具有六方结构的有序介孔纳米硅颗粒 和介孔二氧化硅钝掺合纳米颗粒造影剂. 由此可见, 组 合化学技术在开发纳米材料库过程中表现出巨大优势,
大大加快了寻找目标纳米材料的速度.

\section{4 高通量技术在合成单层石墨烯中的应用}

石墨烯具有良好的载流子迁移率、导热性、高强度 和超大的比表面积. 这些优异的性能使得石墨烯在纳米 电子器件、气体传感器和能量存储等领域有很好的应用 前景 ${ }^{[27,28]}$. 但是, 对制备出单层石墨烯和批量处理石墨 烯样品还存在很多困难 ${ }^{[29]}$, 近年来, 研究人员提出了利 用高通量技术来加工合成单层石墨烯 ${ }^{[30,31]}$. Tung 等 ${ }^{[30]}$ 利 用纯肼来消除石墨烯氧化物的氧化功能并使其恢复一 维平面结构得到单层石墨烯. 实验得到的大表面积石墨 烯使得其后期加工更简易化，可以高通量实现石墨烯的 加工处理. Ang 等 ${ }^{[31]}$ 利用一种高通量插层剥离方法从弱 氧化、低分散性的石墨烯氧化物中制备出单层石墨烯, 产量高达 $90 \%$ 多.

\section{3 组合化学在纳米生物医学中的应用}

近年来, 利用组合化学来构建纳米材料表面化学性 质多样性也是纳米表面化学的研究热点之一. 随着纳米 科技的迅速发展，其在生物医学领域的应用越来越广 泛. 一些纳米材料由于其特殊的理化性质成为潜在的新 型药物载体和应用于分子成像诊断领域. 但是, 许多纳 米材料由于具有相对较低的水溶性以及较高的生物毒 性等缺点, 大大限制了它们在生物医学领域的应用. 由 于纳米粒子具有较大的比表面积，人们希望通过对纳米 材料表面进行化学修饰, 改变其表面化学性质来调节它 的生物活性，从而提高其在生物医学领域的应用价值. 一方面, 可以利用组合化学技术在纳米粒子表面引入一 些基团来提高它们的生物相容性，如提高纳米粒子的分 散性、降低纳米粒子的生物毒性等; 另一方面, 可以加 入一些特定小分子来增强它对肿瘤细胞的识别性和靶 向性, 提高癌症的早期诊断与治疗能力等.

\section{1 提高纳米材料的生物相容性}

\subsection{1 提高纳米材料的稳定性}

利用常规方法合成一些纳米颗粒的时候, 由于纳米 颗粒表面吸附了疏水性物质而导致纳米颗粒在水溶液 中的分散性不好或者不能进行修饰功能化 ${ }^{[36,37]}$. 如果没 有适当的稳定剂, 表面化学高度敏感的纳米材料会自发 的发生团聚而不稳定. 在纳米颗粒表面修饰上一些水溶 性聚合物、氨基酸和壳聚糖等亲水性基团是提高纳米颗 粒稳定性最直接的方法 ${ }^{[38,39]}$. Lévy 等 ${ }^{[40]}$ 前期利用五肽分 子 CALNN 来修饰纳米金, 研究结果发现这个五肽能够 有效提高金纳米粒子的稳定性. 随后，他们又设计了 58 种具有类似结构的多肽, 并利用它们修饰纳米金建立了 一个金纳米库，通过加入 $\mathrm{NaCl}$ 或调节 $\mathrm{pH}$ 来研究这几十 种纳米金粒子在水溶液中的稳定性(图 2). 实验结果表 明, 多肽链长度的增长以及多肽序列中的颈基都有助于 提高金纳米颗粒抗盐能力, 同时改变肽段中疏水和亲水 
的氨基酸种类也会对金纳米的稳定性有很大影响.

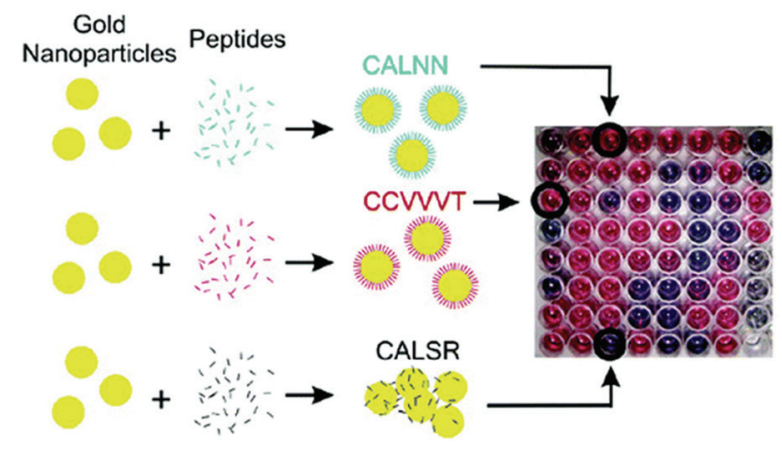

图 2 利用表面修饰化调节金纳米颗粒的稳定性. 从参考文献[40]允 许转载, 2004 American Chemical Society 版权所有

Figure 2 Regulation of the stabilities of gold nanoparticles by surface modification

\subsection{2 降低纳米材料的生物毒性}

大量研究表明一些纳米材料对小鼠具有一定的毒 性 ${ }^{[41 \sim 43]}$, 且对人体的巨噬细胞 ${ }^{[4]}$ 、肺上皮细胞 ${ }^{[4]}$ 、角质 细胞 ${ }^{[46]}$ 和免疫细胞 ${ }^{[47]}$ 等均可产生细胞毒效应, 这些毒 性效应大大限制了它的应用范围。研究表明, 通过表面 修饰功能化可以有效的调控纳米材料的生物活性 ${ }^{[26,48]}$. 我们在前期研究中, 证明了羧基化碳纳米管可以通过影 响 BMP 信号通路产生细胞周期阻滞, 影响细胞功能 ${ }^{[49]}$. 为了得到生物相容性更好的多壁碳纳米管(MWCNTs), 我们利用组合化学技术合成了含 80 种不同表面化学性 质的 MWCNTs 库 ${ }^{[48]}$. 根据蛋白结合实验、细胞毒性评 价以及免疫响应干扰实验, 综合评价库中的多壁碳纳米 管的生物相容性(图 3). 实验结果表明, 一些表面修饰基 团可以有效降低 MWCNTs 的细胞毒性和免疫毒性, 综 合评价结果显示库中的 45 号、49 号、57 号以及 65 号 MWCNTs 的生物相容性得到了最大提高.

为了证明对碳纳米管进行表面修饰可以提高它的 分子识别能力, 我们又进一步对库中的 MWCNTs 和糜 蛋白酶 $(\mathrm{ChT})$ 作用的催化位点和荧光淬灭位点进行了篮 选实验. 研究结果表明, 库中的 30 号、34 号、35 号和 36 号 MWCNTs 可以特异性结合在糜蛋白酶的催化位点 上而几乎不与酶的苂光淬灭位点结合, 竞争性抑制了 $\mathrm{ChT}$ 的活性 ${ }^{[50]}$.

另外, 为了探讨各个碳纳米管引起免疫干扰程度的差异 原因, 我们继续选择两种免疫干扰程度差异较大的表面 修饰化 MWCNTs 进行研究 ${ }^{[51]}$, 发现 MWCNTs 的表面修 饰会改变它对清道夫受体的识别能力, 从而干扰 $\mathrm{NF \kappa B}$ 信号通路的激活, 调控免疫毒性. 免疫干扰弱的 MWCNTs 是由于其特定的表面修饰增强了清道夫受体 的识别, 减少 NFkB 信号通路的激活, 从而降低免疫毒 性.

\section{2 增强细胞靶向性}

在纳米材料上修饰能与细胞表面受体特异性结合

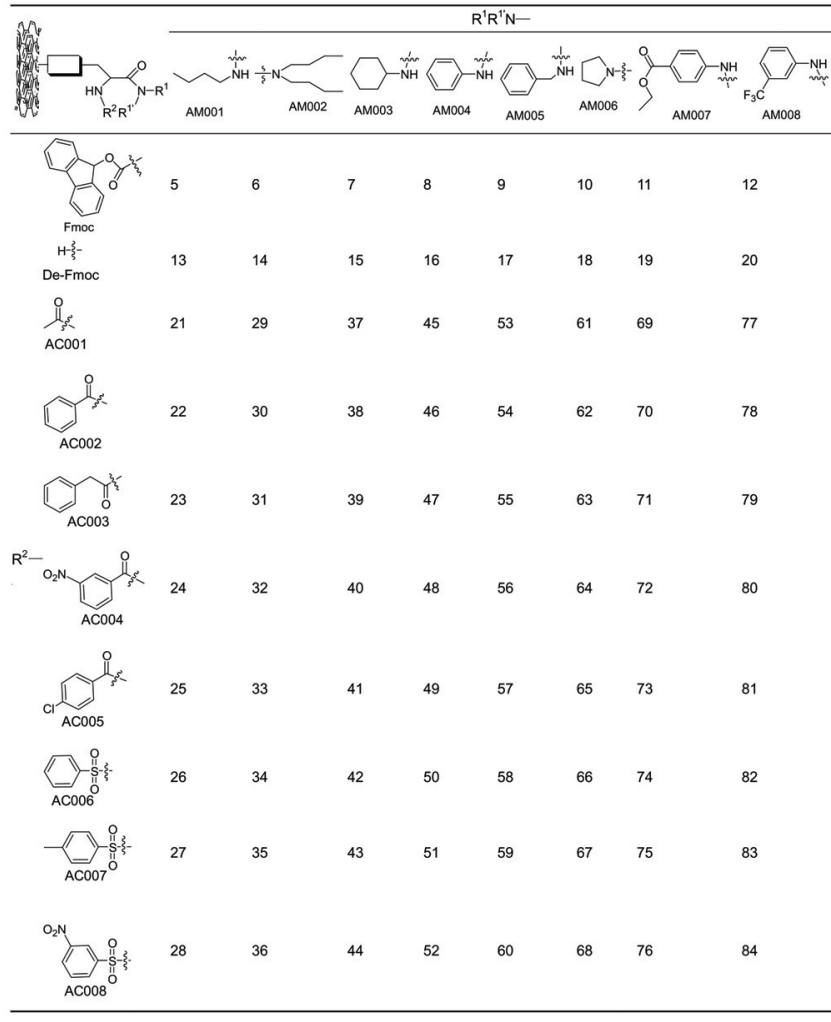

图 3 多壁碳纳米管组合库表面修饰的基团结构. 从参考文献[48]允 许转载, 2008 American Chemical Society 版权所有

Figure 3 Surface molecular compositions of combinatorial MWNT library members

的配体可以有效增强纳米材料对特定细胞的靶向性. 例 如，可以通过在磁性氧化铁纳米粒子上共价修饰上抗 体、多肽和一些小分子来控制它的靶向性 ${ }^{[52]}$. Weissleder 等 ${ }^{[53]}$ 在一个表面有 62 个自由氨基的交联氧化铁纳米颗 粒(CLIO)修饰上少量近红外荧光染料 Cy5.5, 同时选择 146 种不同的小分子基团(MW $<500 \mathrm{Da})$ 共价连接到剩 余的自由氨基上得到一个组合 CLIO 库, 并通过在水中 的稳定性和生物活性实验对它们进行篎选. 笁选实验结 果显示, 库中的一些 CLIO 纳米粒子对内皮细胞、活化 的人类巨噬细胞或者胰腺癌细胞具有高度的特异性. 另 外, Leimgruber 等 ${ }^{[54]}$ 发现 CLIO 在修饰前能够被所有类 型的巨噬细胞所识别摄取, 当在 CLIO 上修饰甘氨酸, 通过对多种巨噬细胞系篎选结果得到一种甘氨酸-CLIO 纳米颗粒对具有促血管生长和免疫抑制特点的肿瘤巨 噬细胞具有特异性靶向能力.

上述结果主要是针对巨噬细胞, 后续的一些研究可 以更进一步证明上述方法的可行性. Kelly 等 ${ }^{[55]}$ 从多个 血管床中分离出多个初级人类内皮细胞, 对它们进行笛 选. 利用严格解析方法对多维数据进行分析. 管选结果 经统计计算后, 以热图形式表现, 研究发现其中有四种 磁性纳米粒子对 11 种人类内皮细胞具有强靶向性但对 两种巨噬细胞的靶向性很弱(图 4). 


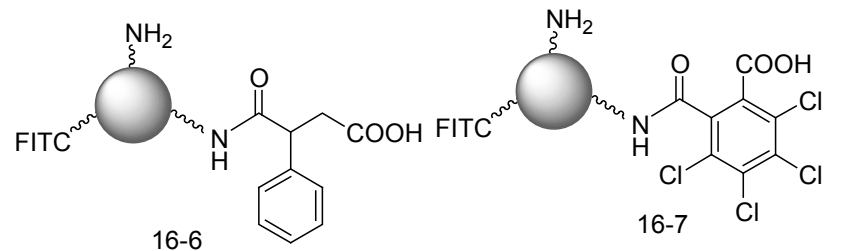<smiles>N[C@H]1C[C@H](C(F)F)CC[C@H]1NC(=O)CC1(CC(=O)O)CCCC1</smiles>

图 4 四种表面不同修饰功能化的磁性纳米颗粒

Figure 4 Four magnetic nanoparticles with different surface chemistry

另外, 纳米材料与传统的诊断造影剂相比具有体积 小、生物相容性好和器官靶向能力强等优势, 为生物医 学成像研究提供了一个新的平台. 纳米核磁共振成像技 术可以显著提高肿瘤诊断的精密度与准确度. Lee 等 ${ }^{[56]}$ 利用氧化铁纳米颗粒建立了一种超灵敏的 MRI 纳米探 针, 他们在氧化铁纳米颗粒表面修饰上曲妥单抗, 曲妥 单抗是一种能与肿瘤细胞特异性结合的抗体, 从而增强 了肿瘤细胞的检测灵敏度. 这种探针在体内能检测到的 肿瘤最小能达到 $50 \mathrm{mg}$. Qian 等 ${ }^{[57}$ 设计了一种能与肿瘤 细胞表面的 EGR 受体特异性结合的单链片段抗体, 利 用抗体修饰化的纳米金颗粒通过表面增强拉曼光谱来 提高肿瘤成像. 上述研究结果说明了对纳米材料表面进 行特定修饰功能化可以有效提高它们的肿瘤细胞靶向 性, 从而提高它们的癌症诊断能力.

由于目前已知的能用于增强靶向性的肿瘤相关蛋 白种类不多, 有大量与识别肿瘤细胞相关的研究工作需 要被探讨, 而组合化学策略和高通量技术可以有效推进 完成这项工作. 例如, 可以通过在纳米材料表面修饰第 二种能与细胞表面的其他受体特异性结合的配体来增 强纳米材料对癌症细胞的识别, 这种方法对存在个体差 异的一些癌症治疗方面非常有用. 我们在前期利用组合 化学方法合成了一个双配体修饰的多功能化金纳米 库 ${ }^{[58]}$, 其中第一种配体固定为叶酸分子, 用于对叶酸受 体过表达的癌细胞的识别(图 5a). 通过改变第二种配体 的结构合成出多功能化金纳米库, 利用多种癌细胞系 (Hela 细胞、 $\mathrm{KB}$ 细胞以及 $\mathrm{HepG} 2$ 细胞) 对金纳米库进行 细胞识别篎选实验. 研究结果表明, 一些双配体修饰的 金纳米与单配体修饰的金纳米相比, 其对细胞的识别能 力有明显提高, 最高可达 $4 \sim 5$ 倍(图 $5 b \sim 5 \mathrm{~d}$ ). 因此, 这 些优化的纳米粒子在实现药物传送、疾病诊断和放射治 疗过程中可以有效提高对肿瘤的靶向, 减少对正常细胞 的损伤.

\section{4 用于纳米技术研究中的高通量篮选技术}

高通量篮选最初是伴随着组合化学而产生的一种
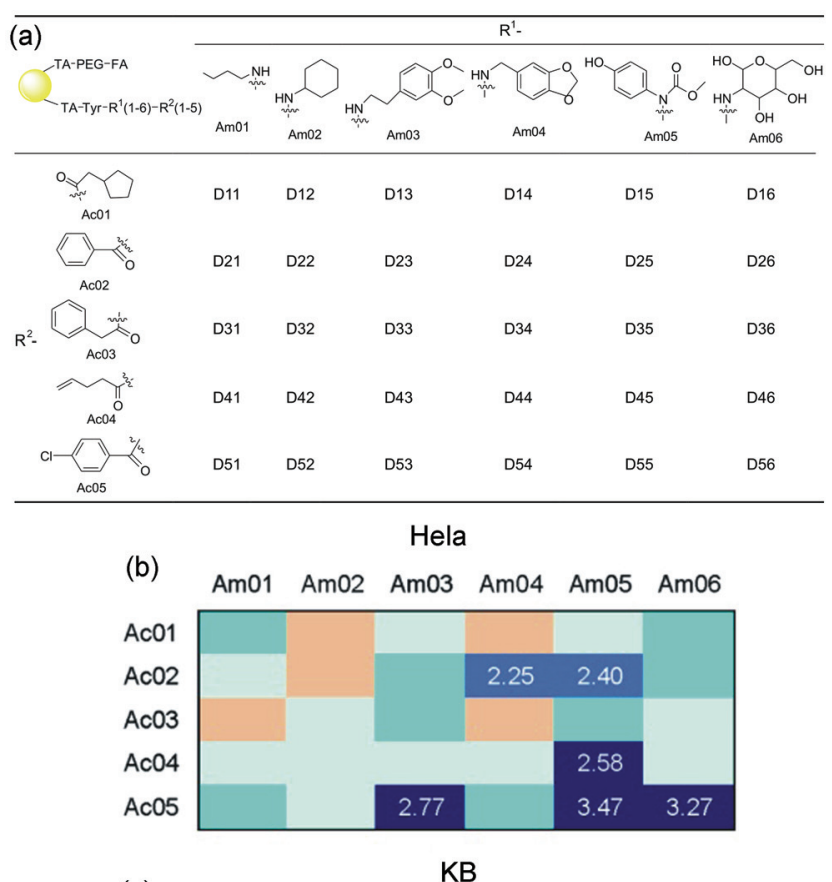

(c) 1 KB

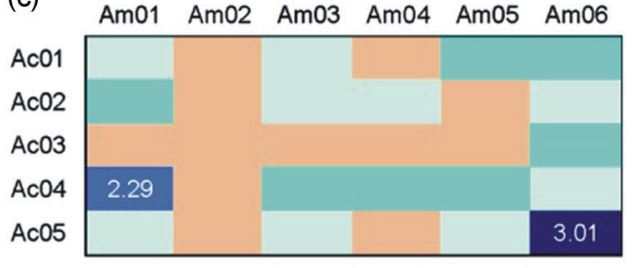

(d) $\begin{gathered}\text { HepG2 } \\ \text { Am01 Am02 Am03 Am04 Am05 Am06 }\end{gathered}$

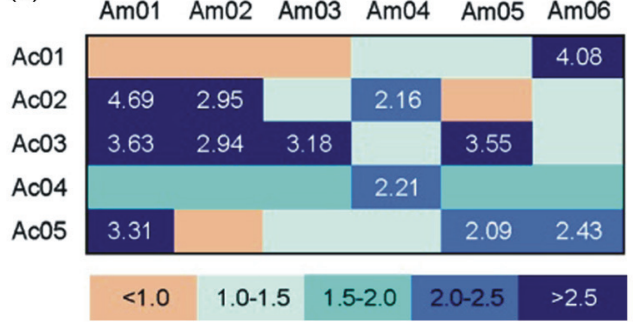

图 5 (a)修饰化金纳米库的表面基团结构; (b d ) 不同细胞系对双配 体修饰化金纳米库摄取结果的热图; (b) Hela 细胞、(c) KB 细胞以及 (d) HepG2 细胞. 实验中所用金纳米浓度为 $50 \mu \mathrm{g} / \mathrm{mL}$. 从参考文献[58]允 许转载, 2011 American Chemical Society 版权所有

Figure 5 (a) Surface molecular compositions of dual-ligand GNP array members. $(b \sim d)$ Heat maps showing the relative cellular uptake amounts of dual-ligand GNPs for (b) Hela, (c) KB, and (d) HepG2 cells in comparison with GNP-FA. The GNP concentration for all experiments was 50 $\mu \mathrm{g} / \mathrm{mL}$

药物笁选方式, 它可以在短时间内对大量候选化合物完 成篮选. 高通量篮选技术对于在一个庞大的组合纳米材 料库中快速篮选出目标纳米粒子以及如何同时进行大 量笁选实验是一个重要突破. 随着纳米组合化学的诞 生，越来越多的高通量篮选技术在纳米技术研究中得到 应用。

磁共振成像(Magnetic Resonance Imaging, MRI)是 利用原子核在磁场内共振所产生信号经重建成像的一 
种成像技术. 自 20 世纪 80 年代作为一种医学新技术被 应用以来，它以极快的速度得到发展. 近年来也被应用 于纳米高通量篮选, 主要用于篮选磁性纳米材料 ${ }^{[59 ~ 61]}$. Hogemann 等 ${ }^{[60]}$ 利用 MRI 作为一种高通量篎选技术研究 超顺磁纳米材料与细胞相互作用, 并用于表面多肽修饰 化纳米材料库的细胞靶向性以及靶向位点的鉴定(图 6). Acharya 等 ${ }^{[61]}$ 在脂质立方液晶纳米粒中负载上超顺磁纳 米颗粒，利用 MRI 进行高通量篮选发现，这个混合中间 相纳米粒子的 $\mathrm{T} 2$ 加权成像效果明显提高, 并且随着负 载的超顺磁纳米颗粒比例的增加, $\mathrm{T} 2$ 加权成像效果增加 越明显. 因此, MRI 高通量篎选技术可以大大促进特定 细胞靶向的磁共振纳米造影剂的发展.

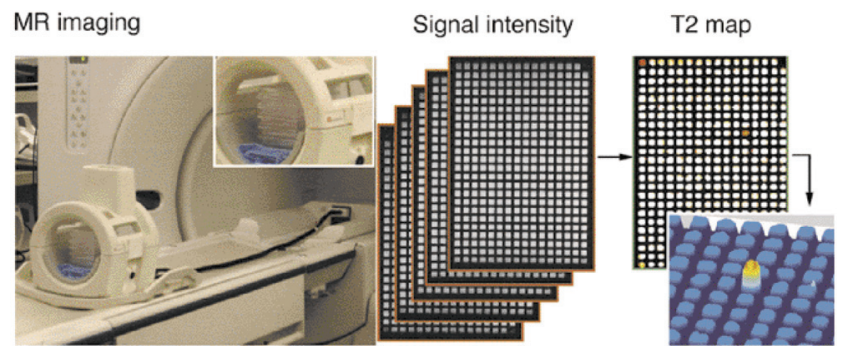

图 6 利用 MR 成像技术进行高通量篮选. 每个微孔板在发射不同脉 冲序列的正交线圈中成像, 产生一系列图像. 将信号强度进行处理形 成一个 T2 图来评价结果. 从参考文献[60]允许转载, 2002 American Chemical Society 版权所有

Figure 6 Use of a MR imager for high throughput screening. Microtiter plates were imaged in a quadrature coil with different pulse sequences (variable TE) to yield a series of images for each plate. Signal intensity data were processed to yield a T2 map indicating wells with "hits"

Haun 等 ${ }^{[62]}$ 建立了一种能够快速量化剖析多个标记 蛋白的临床微核磁设备( $\mu \mathrm{NMR})$ 技术. 并且利用 $\mu \mathrm{NMR}$ 对 50 个病患, 选择 9 种肿瘤细胞表面标记物进行研究. 首先从病患体内提取出疑似人类上腹部恶性肿瘤细胞, 在细胞上标记一个能与细胞表面标志物特异性结合的 反式环辛烯修饰化的抗体, 然后再标记上能与抗体特异 性结合的四嗪修饰化的磁性纳米粒子. 通过磁性纳米粒 子的磁性能, 利用 $\mu \mathrm{NMR}$ 进行篮选. 研究结果发现, 样 品的 $\mu$ NMR 信号与标准化临床测定结果(如流式、免疫 组化等)具有高度相关性. 但是, 标准化临床测定需要 $3 \sim 4 \mathrm{~d}$ 的时间, 而 $\mu \mathrm{NMR}$ 的测定只需要不到一小时的时 间, 大大提高临床病理诊断效率.

自动化基因芯片系统是一种能对细胞的基因表达 进行高通量检测与分析技术. Zhang 等 $^{[63]}$ 用这种技术来 高通量篎选不同浓度的 PEG 修饰化的硅烷化 CdSe/ZnS 核壳量子点对皮肤上皮细胞基因表达的影响. Ding 等 ${ }^{[64]}$ 利用基因组表达阵列分析和高含量表现成像分析来比 较不同浓度的多壁碳纳米圈(MWCNOs)和 MWCNTs 引 起人类皮肤成纤维细胞不同的毒性机制. 研究结果发 现, MWCNTs 主要是改变了细胞内免疫和炎症相关基 因的表达, 而 MWCNOs 主要是改变外界刺激引起响应 的相关基因的表达.
荧光激活细胞分类术(Fluorescent Activated Cell Sorter，FACS) 是一种高通量的细胞笕选技术，它利用流 式细胞仪高灵敏度、高通量等优点, 能以极高的速度 $(>$ 108/天)对细胞系进行师选. 当研究有苂光的纳米材料 与细胞之间的相互作用时, 也可以利用 FACS 技术来进 行定量研究, 一些研究者已经利用此技术来对苂光纳米 材料库进行篎选 ${ }^{[65,66]}$. Lorenz 等 ${ }^{[65]}$ 利用 FACS 技术研究 了多种细胞系对一系列带有不同表面电荷的荧光聚合 物纳米颗粒的摄取情况. Schellenberger 等 ${ }^{[66]}$ 平行合成了 一个不同表面修饰的荧光超顺磁氧化铁纳米颗粒库, 通 过 FACS 分析技术和荧光显微技术来篮选库中的纳米颗 粒对正常和调亡细胞的识别能力.

另外，高通量发光菌法不仅可以用来评价分析一些 环境物质和其它化合物的毒性，还可以应用于纳米材料 的毒性篎选 ${ }^{[0]}$. $X$ 射线小角散射技术已被用于磁共振造 影剂的介孔二氧化硅库的体外笁选 ${ }^{[35]}$. 低数值孔径光 热显微技术近年来作为一种具有潜力的高通量篮选技 术而被用于金纳米库的篎选 ${ }^{[64]}$.

\section{5 总结与展望}

利用组合化学技术不仅在短时间内可以建立起一 个庞大的不同表面化学修饰的功能化纳米材料库, 还可 以通过高通量实现库中纳米材料的快速篎选. 它的高效 性将为纳米材料的开发和应用前景带来强大的推动力. 但是, 在纳米技术研究领域, 组合化学还处于一个新兴 状态, 在很多研究方面需要不断地被完善, 例如以往对 纳米表面功能化研究较多的是通过增强纳米材料的靶 向性来提高其生物医学应用价值, 但在实际应用中需要 考虑的因素还有很多, 比如生物相容性、良好的药物动 力学、吸收、分布、代谢等; 纳米材料的计算机建模和 复杂的高通量篮选对纳米组合化学技术来说也是巨大 的挑战. 另外, 纳米组合技术面临的另外一个挑战是表 征纳米材料表面性质方法学的建立, 主要是对纳米材料 表面化学修饰分子结构的识别以及结合量的定量化. 因 此, 未来还需要进行大量的实验进行优化研究, 组合化 学方法在纳米研究方面将会呈现出更大的优势.

\section{作者简介}

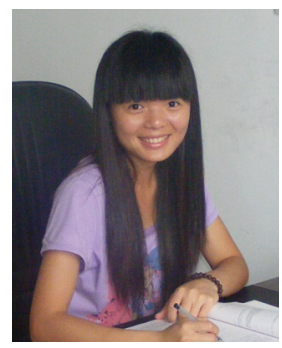

吴金梅, 女, 1987 年出生, 硕士研究生, 主要从事纳米材 料与生物分子相互作用的研究. 


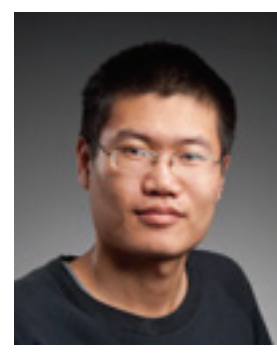

苏高星, 男, 1986 年出生, 博士研究生, 主要从事纳米组 合化学合成功能化纳米材料的研究.

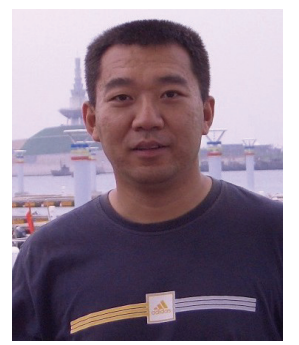

张斌, 男, 1968 年出生, 博士, 教授, 硕士生导师, 主要从 事纳米分析化学的研究.

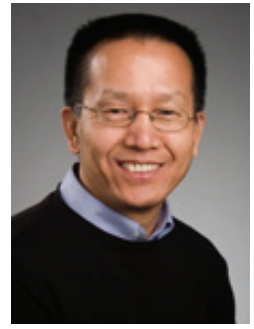

间兵, 男, 1957 年出生, 博士, 教授, 博士生导师, 主要从 事组合化学、纳米分析与毒理学的研究.

\section{References}

[1] Gordon, E. M.; Barrett, R. W.; Dower, W. J.; Fodor, S. P. A.; Gallop, M. A. J. Med. Chem. 1994, 37, 1385.

[2] Duan, S.; Kahn, M.; Senkan, S. Comb. Chem. High T. Scr. 2007, 10, 111.

[3] Green, J. J.; Langer, R.; Anderson, D. G. Acc. Chem. Res. 2008, 41, 749.

[4] Lindell, S. D.; Pattenden, L. C.; Shannon, J. Bioorg. Med. Chem. 2009, 17, 4035 .

[5] Ferrari, M. Nat. Rev. Cancer 2005, 5, 161.

[6] Youtie, J.; Iacopetta, M.; Graham, S. J. Technol. Tran. 2008, 33, 315.

[7] Su, G.; Yan, B. J. Comb. Chem. 2010, 12, 215.

[8] Hermans, S.; Raja, R.; Thomas, J. M.; Johnson, B. F. G.; Sankar, G.; Gleeson, D. Angew. Chem., Int. Ed. 2001, 40, 1211.

[9] Glaspell, G.; Fuoco, L.; El-Shall, M. S. J. Phys. Chem. B 2005, 109, 17350.

[10] Son, S. U.; Jang, Y.; Park, J.; Na, H. B.; Park, H. M.; Yun, H. J.; Lee, J.; Hyeon, T. J. Am. Chem. Soc. 2004, 126, 5026.

[11] Senkan, S.; Kahn, M.; Duan, S.; Ly, A.; Leidholm, C. Catal. Today 2006, 117, 291.

[12] Kahn, M.; Seubsai, A.; Onal, I.; Senkan, S. Comb. Chem. High T. Scr. 2010, 13, 67.

[13] Noda, S.; Tsuji, Y.; Murakami, Y.; Maruyama, S. Appl. Phys. Lett. 2005, 86, 173106.

[14] Noda, S.; Sugime, H.; Osawa, T.; Tsuji, Y.; Chiashi, S.; Murakami, Y.; Maruyama, S. Carbon 2006, 44, 1414.
[15] Ago, H.; Nakamura, K.; Imamura, S.; Tsuji, M. Chem. Phys. Lett. 2004, 391, 308

[16] Dai, H.; Rinzler, A. G.; Nikolaev, P.; Thess, A.; Colbert, D. T.; Smalley, R. E. Chem. Phys. Lett. 1996, 260, 471

[17] Vegas, A. J.; Anderson, D. G. Polym. Sci.: A Compr. Ref. 2012, 9, 457.

[18] Zugates, G. T.; Tedford, N. C.; Zumbuehl, A.; Jhunjhunwala, S.; Kang, C. S.; Griffith, L. G.; Lauffenburger, D. A.; Langer, R.; Anderson, D. G. Bioconjugate Chem. 2007, 18, 1887.

[19] Van Vliet, L. D.; Chapman, M. R.; Avenier, F.; Kitson, C. Z.; Hollfelder, F. ChemBioChem 2008, 9, 1960.

[20] Akinc, A.; Zumbuehl, A.; Goldberg, M.; Leshchiner, E. S.; Busini, V.; Hossain, N.; Bacallado, S. A.; Nguyen, D. N.; Fuller, J.; Alvarez, R. Nat. Biotechnol. 2008, 26, 561.

[21] Anderson, D. G.; Lynn, D. M.; Langer, R. Angew. Chem., Int. Ed. 2003, 115, 3261 .

[22] Sunshine, J. C.; Akanda, M. I.; Li, D.; Kozielski, K. L.; Green, J. J. Biomacromolecules 2011, 12, 3592 .

[23] Anderson, K.; Sizovs, A.; Cortez, M.; Waldron, C.; Haddleton, D. M.; Reineke, T. M. Biomacromolecules 2012, 13, 2229.

[24] Sunshine, J. C.; Peng, D. Y.; Green, J. J. Mol. Pharm. 2012, 9, 3375.

[25] Martello, F.; Piest, M.; Engbersen, J. F. J.; Ferruti, P. J. Controlled Release 2012, 164, 372.

[26] Barua, S.; Joshi, A.; Banerjee, A.; Matthews, D.; Sharfstein, S. T.; Cramer, S. M.; Kane, R. S.; Rege, K. Mol. Pharm. 2008, 6, 86.

[27] Zhang, Y.; Tan, Y. W.; Stormer, H. L.; Kim, P. Nature 2005, 438, 201.

[28] Lee, C.; Wei, X.; Kysar, J. W.; Hone, J. Science 2008, 321, 385.

[29] Viculis, L. M.; Mack, J. J.; Kaner, R. B. Science 2003, 299, 1361.

[30] Tung, V. C.; Allen, M. J.; Yang, Y.; Kaner, R. B. Nat. Nanotechnol. 2008, 4, 25.

[31] Ang, P. K.; Wang, S.; Bao, Q.; Thong, J. T. L.; Loh, K. P. ACS Nano 2009, 3, 3587.

[32] Alexander, K. D.; Hampton, M. J.; Zhang, S.; Dhawan, A.; Xu, H.; Lopez, R. J. Raman Spectrosc. 2009, 40, 2171.

[33] Mulet, X.; Kennedy, D. F.; Conn, C. E.; Hawley, A.; Drummond, C. J. Int. J. Pharm. 2010, 395, 290.

[34] Han, S. M.; Shah, R.; Banerjee, R.; Viswanathan, G.; Clemens, B.; Nix, W. Acta Mater. 2005, 53, 2059.

[35] Tse, N. M. K.; Kennedy, D. F.; Moffat, B. A.; Kirby, N.; Caruso, R. A.; Drummond, C. J. ACS Comb. Sci. 2012, 14, 443.

[36] Li, J. J.; Wang, Y. A.; Guo, W.; Keay, J. C.; Mishima, T. D.; Johnson, M. B.; Peng, X. J. Am. Chem. Soc. 2003, 125, 12567.

[37] Jana, N. R.; Peng, X. J. Am. Chem. Soc. 2003, 125, 14280.

[38] Sheng, Y.; Liu, C.; Yuan, Y.; Tao, X.; Yang, F.; Shan, X.; Zhou, H.; Xu, F. Biomaterials 2009, 30, 2340.

[39] Basiruddin, S.; Saha, A.; Pradhan, N.; Jana, N. R. J. Phys. Chem. C 2010, 114, 11009.

[40] Lévy, R.; Thanh, N. T. K.; Doty, R. C.; Hussain, I.; Nichols, R. J.; Schiffrin, D. J.; Brust, M.; Fernig, D. G. J. Am. Chem. Soc. 2004, 126,10076

[41] Dobrovolskaia, M. A.; Germolec, D. R.; Weaver, J. L. Nat. Nanotechnol. 2009, 4, 411.

[42] Hauck, T. S.; Anderson, R. E.; Fischer, H. C.; Newbigging, S.; Chan, W. C. W. Small 2009, 6, 138.

[43] Pisanic, T. R.; Blackwell, J. D.; Shubayev, V. I.; Fiñones, R. R.; Jin, S. Biomaterials 2007, 28, 2572.

[44] Cheng, C.; Müller, K. H.; Koziol, K. K. K.; Skepper, J. N.; Midgley, P. A.; Welland, M. E.; Porter, A. E. Biomaterials 2009, 30, 4152.

[45] Lanone, S.; Rogerieux, F.; Geys, J.; Dupont, A.; Maillot-Marechal, E.; Boczkowski, J.; Lacroix, G.; Hoet, P. Part. Fibre Toxicol. 2009, 6,455 .

[46] Rouse, J. G.; Yang, J.; Barron, A. R.; Monteiro-Riviere, N. A. Toxicol. in Vitro 2006, 20, 1313.

[47] Hanley, C.; Thurber, A.; Hanna, C.; Punnoose, A.; Zhang, J.; Wingett, D. G. Nanoscale Res. Lett. 2009, 4, 1409.

[48] Zhou, H. Y.; Mu, Q. X.; Gao, N. N.; Liu, A. F.; Xing, Y. H.; Gao, S. L.; Zhang, Q.; Qu, G. B.; Chen, Y. Y.; Liu, G.; Zhang, B.; Yan, B. Nano Lett. 2008, 8, 859.

[49] Mu, Q. X.; Du, G.; Chen, Y. Y.; Zhang, B.; Yan, B. ACS Nano 2009, 3, 1139.

[50] Zhang, B.; Xing, Y.; Li, Z.; Zhou, H.; Mu, Q.; Yan, B. Nano Lett. 2009, 9, 2280.

[51] Gao, N.; Zhang, Q.; Mu, Q.; Bai, Y.; Li, L.; Zhou, H.; Butch, E. R.; Powell, T. B.; Snyder, S. E.; Jiang, G.; Yan, B. ACS Nano 2011, 5 , 4581. 
[52] McCarthy, J. R.; Weissleder, R. Adv. Drug Delivery Rev. 2008, 60, 1241.

[53] Weissleder, R.; Kelly, K.; Sun, E. Y.; Shtatland, T.; Josephson, L. Nat. Biotechnol. 2005, 23, 1418 .

[54] Leimgruber, A.; Berger, C.; Cortez-Retamozo, V.; Etzrodt, M.; Newton, A. P.; Waterman, P.; Figueiredo, J. L.; Kohler, R. H.; Elpek, N.; Mempel, T. R. Neoplasia (New York, NY) 2009, 11, 459.

[55] Kelly, K. A.; Shaw, S. Y.; Nahrendorf, M.; Kristoff, K.; Aikawa, E.; Schreiber, S. L.; Clemons, P. A.; Weissleder, R. Integr. Biol. 2009, 1,311 .

[56] Lee, J. H.; Huh, Y. M.; Jun, Y.; Seo, J.; Jang, J.; Song, H. T.; Kim, S.; Cho, E. J.; Yoon, H. G.; Suh, J. S.; Cheon, J. Nat. Med. 2007, 13, 95.

[57] Qian, X.; Peng, X. H.; Ansari, D. O.; Yin-Goen, Q.; Chen, G. Z.; Shin, D. M.; Yang, L.; Young, A. N.; Wang, M. D.; Nie, S. Nat. Biotechnol. 2007, 26, 83 .

[58] Zhou, H. Y.; Jiao, P. F.; Yang, L.; Li, X.; Yan, B. J. Am. Chem. Soc. 2010, 133, 680.

[59] Muir, B. W.; Moffat, B. A.; Harbour, P.; Coia, G.; Zhen, G.; Waddington, L.; Scoble, J.; Krah, D.; Thang, S. H.; Chong, Y. K. J. Phys. Chem. C 2009, 113, 16615.
[60] Högemann, D.; Ntziachristos, V.; Josephson, L.; Weissleder, R. Bioconjugate Chem. 2002, 13, 116.

[61] Acharya, D. P.; Moffat, B. A.; Polyzos, A.; Waddington, L.; Coia G.; Wright, D. K.; Wang, H. X.; Egan, G. F.; Muir, B. W.; Hartley, P. G. RSC Adv. 2012, 2, 6655.

[62] Haun, J. B.; Castro, C. M.; Wang, R.; Peterson, V. M.; Marinelli, B S.; Lee, H.; Weissleder, R. Sci. Transl. Med. 2011, 3, 71ra16.

[63] Zhang, T.; Stilwell, J. L.; Gerion, D.; Ding, L.; Elboudwarej, O.; Cooke, P. A.; Gray, J. W.; Alivisatos, A. P.; Chen, F. F. Nano Lett. 2006, 6, 800 .

[64] Ding, L.; Stilwell, J.; Zhang, T.; Elboudwarej, O.; Jiang, H.; Selegue, J. P.; Cooke, P. A.; Gray, J. W.; Chen, F. F. Nano Lett. 2005, 5, 2448.

[65] Lorenz, M. R.; Holzapfel, V.; Musyanovych, A.; Nothelfer, K.; Walther, P.; Frank, H.; Landfester, K.; Schrezenmeier, H.; Mailänder, V. Biomaterials 2006, 27, 2820.

[66] Schellenberger, E. A.; Reynolds, F.; Weissleder, R.; Josephson, L. ChemBioChem 2004, 5, 275. 